\title{
CSF biomarker profiles in CNS infection associated with HSV and VZV mimic pattern in Alzheimer's disease.
}

Makiko Shinomoto

Kyoto Prefectural University of Medicine

TAKASHI KASAI ( $\sim$ kasaita@koto.kpu-m.ac.jp)

Kyoto Prefectural University of Medicine Ishoku Saisei Gekagaku https://orcid.org/0000-0002-6424-0738

Harutsugu Tatebe

National Institutes for Quantum and Radiological Science and Technology

Fukiko Kitani-Morii

Kyoto Prefectural University of Medicine

Takuma Ohmichi

Kyoto Prefectural University of Medicine

Yuzo Fujino

Kyoto Prefectural University of Medicine

\section{David Allsop}

Lancaster University

Toshiki Mizuno

Kyoto Prefectural University of Medicine

\section{Takahiko Tokuda}

National Institutes for Quantum and Radiological Science and Technology

\section{Research}

Keywords: herpes virus, herpes simplex virus, varicella zoster virus, Alzheimer's disease, biomarkers, CSF

Posted Date: September 1st, 2020

DOI: https://doi.org/10.21203/rs.3.rs-66188/v1

License: (c) (i) This work is licensed under a Creative Commons Attribution 4.0 International License. Read Full License 


\section{Abstract}

Background: Central nervous system (CNS) infections have been reported to have a certain etiological relevance to Alzheimer's disease (AD). In particular, herpes simplex virus (HSV) and varicella zoster virus (VZV) infections has been reported as risk factors for AD. The aim of this study was to determine whether or not AD-related biomarkers were changed in patients with HSV or VZV CNS infections.

Methods: Nine patients with HSV infection of the CNS, eight patients with VZV complicated by CNS involvement, and eighteen age-matched controls were enrolled. Amyloid $\beta(A \beta)_{1-42}, A \beta_{1-40}$, total-tau (t-tau), tau phosphorylated at threonine 181 (p-tau), neurofilament light chain ( $\mathrm{NfL}$ ), phosphorylated neurofilament heavy chain (p-NfH), glial fibrillary acidic protein (GFAP), and soluble triggering receptor expressed on myeloid cells 2 (sTREM2) in were measured in cerebrospinal fluid (CSF), and NfL in serum.

Results: Compared with the control group, CSF $A \beta_{1-42}, A \beta_{1-40}$, and the $A \beta_{1-42} / A \beta_{1-40}$ ratio were significantly decreased, and CSF t-tau, p-tau, sTREM2, and GFAP were significantly increased in the HSV and VZV combined group, in which biomarker changes were similar to those reported in AD. CSF NfL levels measured on admission were significantly correlated with the disease severity and a poor outcome after age adjustment. Serum NfL on admission was also associated with disease severity after age adjustment.

Conclusions: The fact that the biomarker profile in patients with CNS HSV and VZV infections mimicked that in AD patients should be paid attention to as a potential confounding factor in CSF biomarker-based diagnosis of $A D$, and it suggests an etiological similarity between herpetic virus infection and AD. The CSF NfL concentration on admission may be useful as a predictive marker of severity and prognosis in patients with CNS HSV and VZV infections.

\section{Background}

Alzheimer's disease (AD) is the most common cause of dementia. Numerous studies searching for useful ADbiomarkers have reported the value of measuring levels of amyloid $\beta(A \beta)_{1-42}$ as well as total (t-tau) and phosphorylated ( $\mathrm{p}$-tau) tau in cerebrospinal fluid (CSF), or visualizing plaques and tangles by means of positron emission tomography brain imaging using specific probes for aggregated $A \beta$ or tau [1] [2] [3] [4]. The current consensus is that these CSF biomarkers can reflect these AD pathologies at least several years prior to onset and, therefore, can be used to differentiate preclinical AD from normal aging and other dementing disorders [2] [5] [6].

Although AD was initially considered to be a cell autonomous neurodegenerative disorder, marked neuroinflammation is observed in the brains of patients with $A D$, alongside $A \beta$ and tau pathology. Based on genetic and molecular biological findings, central nervous system (CNS) inflammatory processes have been postulated to be involved in the etiopathogenesis of $A D$, in which activated microglia play a key role. This has also been supported by the epidemiological observation that CNS infections were associated with the development of $A D$, and in particular the relationship between herpetic virus and $A D$ has been well-investigated [7]. For example, the presence of anti-herpes simplex virus (HSV) antibody was associated with an elevated risk of developing AD [8]. Moreover, anti-herpetic medication was associated with a reduced risk of dementia in a population-based study [9]. Similar results were also observed in the case of varicella zoster virus (VZV) infections [10] [11].

Taking into consideration the reports above, we hypothesized that the biomarker signature representing AD might be observed in patients with herpetic viral CNS infections as a prognostic biomarker of AD development. In the current 
study, we aimed to determine whether or not the biomarkers related to AD and neurodegeneration were changed in patients with CNS infection by HSV and VZV compared with controls. This study focused on CSF levels of $A \beta_{1-42}$, $A \beta_{1-40}$, total-tau (t-tau), and tau phosphorylated at threonine 181 ( $p$-tau) as molecules representing the AD signature; neurofilament light chain ( $\mathrm{NfL}$ ) and phosphorylated neurofilament heavy chain ( $\mathrm{p}-\mathrm{NfH})$ as indicators of axonal injury; soluble triggering receptor expressed on myeloid cells 2 (sTREM2) as a potential biomarker for microglia activity [12]; and glial fibrillary acidic protein (GFAP) as a biomarker for astrocytic damage. We also measured serum levels of NfL as a blood based biomarker for axonal injury.

\section{Methods}

\section{Study design, ethics statement, and subject recruitment}

All study subjects provided written informed consent before participation and the study protocols were approved by the University Ethics Committee (ERB-G-12, Kyoto Prefectural University of Medicine, Kyoto, Japan). Written informed consent from the participants was obtained when possible and, if not, from the nearest relative. Study procedures were designed and performed in accordance with the Declaration of Helsinki. Diagnosis of CNS HSV infection was conducted in accordance with a published guideline for HSV encephalitis [13]. Diagnosis of VZV infection was based on positive VZV PCR results in CSF, elevation of IgM to VZV in serum and/or CSF, or the coexistence of shingles. Patients presenting with dementia before the onset of CNS infection were excluded. We also excluded patients without pleocytosis in CSF $(\geq 5 / \mu \mathrm{L})$ (i.e., patients presenting with only cutaneous shingles or serum HSV IgM elevation without pleocytosis in CSF were not included in the study). We enrolled 9 patients with HSV infection of the CNS (HSV group: 7 patients with HSV encephalitis, 2 patients with HSV meningitis, and 1 patient with HSV myelitis), 8 patients with herpes zoster complicated by CNS involvement (VZV group: 7 patients with meningitis, 1 patient with myelitis). Eighteen age-matched control patients presenting with neither CNS infection nor dementia were also enrolled (control group). All patients visited Kyoto Prefectural University of Medicine between 1990 and 2019 and underwent lumbar puncture for clinical reasons. CSF samples were collected in polypropylene vials from cases in the morning (from 9 a.m. to 12 a.m.) through a lumbar puncture at the L3/L4 or L4/L5 interspace. Immediately after collection, the samples were cleared by centrifugation at $400 \mathrm{xg}$ for $10 \mathrm{~min}$ at $4^{\circ} \mathrm{C}$. When blood collection was done at the same time as CSF collection, serum was separated by centrifugation for $10 \mathrm{~min}$ at $3,000 \mathrm{~g}$ after the blood was allowed to clot for $15 \mathrm{~min}$ at room temperature in blood collection tubes with clot activator and gel separator (Terumo, Tokyo, Japan), and transferred to polypropylene vials. Fresh samples obtained from the enrolled subjects were immediately stored at $-80^{\circ} \mathrm{C}$ until analysis.

\section{Measurements of CSF and serum biomarkers}

We analyzed CSF and serum samples of the HSV and VZV groups obtained on admission for diagnostic purposes, except for case 1 of the VZV group whose CSF sample was obtained on day 4 due to an inadequate volume of CSF on admission. We measured the concentrations of $A \beta_{1-42}, A \beta_{1-40}$, and t-tau in CSF with reagents from a single lot using the Simoa Human Neurology 3-Plex A assay and the concentrations of NfL in CSF and serum, p-NfH, and GFAP in CSF with reagents from a single lot using the Simoa NF-light assay, Simoa pNF-heavy assay, and Simoa GFAP assay, respectively. All measurements were performed on an HD-1 Simoa analyzer according to the protocol provided by the manufacturer (Quanterix, Lexington, MA, USA). The CSF levels of p-tau181 were analyzed using the originally developed p-tau181 assay on the Simoa platform, reported elsewhere [14]. The CSF sTREM2 levels were quantified by ELISA using a RayBio Human TREM-2 ELISA Kit (RayBiotech, Norcross, GA, USA) in accordance with the manufacturer's instructions and a previous report [15]. 
All samples were analyzed in duplicate on one occasion. All cases and controls were evenly distributed on examination.

\section{Statistics}

The level of significance was set at $\mathrm{P}<0.05$. A comparison between two independent groups was performed using the Mann-Whitney U test, and a comparison among three independent groups was performed using the KruskalWallis test and Dunn's multiple comparison procedure. The Chi-square test was used to evaluate the significance of categorical variables. The uni- and multivariate regression analyses were performed using Spearman's rank correlation coefficient test and ordinal logistic regression model, respectively. Comparison among the groups was performed using GraphPad Prism software (version 8.4.2, GraphPad Software, San Diego, USA). Regression analyses were performed using SPSS for Windows version 23 software (IBM Japan Ltd., Tokyo, Japan).

\section{Results}

The demographic characteristics, diagnosis, CSF profiles, results of viral detection, magnetic resonance imaging (MRI) findings, lowest score of the Glasgow coma scale (GCS) during the hospitalization period, and modified Rankin Scale (mRS) at discharge are summarized in Table 1. There was no significant difference in age $(P=0.8465)$ or sex $(P=0.8586)$ among the HSV, VZV, and control groups. Serum samples were collected from 6 patients of the HSV group, 3 patients of the VZV group, and 6 patients from the control group (shown as cases with asterisks in Table 1). The raw data on biomarkers are presented in the Supplementary Table.

Levels of the biomarkers in each group are summarized in Figure 1. There were four significant differences on multiple comparison among the three groups: First, the levels of CSF t-tau were significantly higher in the HSV than control group $(P=0.0016$, Figure 1D). Second, CSF p-tau levels were significantly elevated in the VZV group $(P=0.0102)$ and HSV $(P=0.0487)$ groups compared with the control group (Figure 1E). Third, the CSF $p$-tau/t-tau ratio was significantly higher in the VZV compared not only with the control but also the HSV group ( $P=0.0389$ and $P=0.0291$, respectively, Figure 1F). Fourth, levels of CSF GFAP were significantly increased in the HSV compared with the control group $(P=0.0202$, Figure $1 \mathrm{~J})$. The HSV and VZV groups trended to have higher CSF sTREM2 and lower CSF $A \beta_{1-42}$ levels compared with the control. However, these trends did not reach significance (Figure $1 \mathrm{l}$ and 1A).

For comparison between herpetic CNS infection and control groups, we combined the data of the HSV and VSV groups, and then compared their biomarker values to those of the control (shown in Figure $2 \mathrm{~A}$ to $2 \mathrm{~K}$ ). In this comparison, we found the following significant differences: The levels of CSF $A \beta_{1-42}, A \beta_{1-40}$, and the $A \beta_{1-42} / A \beta_{1-40}$ ratio were significantly lower in the HSV and VSV combined group (HSV/VSV) compared with the control group ( $P=0.01836, P=0.0380$, and $P=0.0262$, respectively) (Figure $2 A, 2 B$, and $2 C$ ). CSF t-tau, $p$-tau, CSF sTREM2, and CSF GFAP levels were significantly elevated in the HSV/VSV group compared with the control group $(P=0.0043$, $\mathrm{P}=0.0007, \mathrm{P}=0.0030$, and $\mathrm{P}=0.0139$, respectively) (Figure $2 \mathrm{D}, 2 \mathrm{E}, 2 \mathrm{I}$, and $2 \mathrm{~J}$ ). The HSV/VZV group tended to have higher CSF p-tau/t-tau, CSF NfL, CSF p-NfH and serum NfL levels compared with the control. However, these trends did not reach significance (Figure $2 \mathrm{~F}, 2 \mathrm{G}$, and $2 \mathrm{H}$ ).

Results of uni- and multivariate regression analyses between those biomarker values and clinical severity are summarized in Table 2. Scatter plots of those are presented in Figure 3 in cases showing significant correlations on univariate analyses as follows; univariate analyses showed significant negative correlations between the lowest score of GCS during the hospitalization and levels of CSF t-tau (Figure 3A), CSF NfL (Figure 3B), CSF p-NfH (Figure 
3C), and serum NfL (Figure 3D) ( $P<0.001, P=0.014, P=0.027$, and $P=0.007$ respectively). The correlations between GCS and NfL in CSF and serum were still robust after age adjustment ( $P=0.014$ and $P=0.030$, respectively), while significance of the relationship of GCS to CSF t-tau and CSF p-NfH disappeared after age adjustment. The levels of CSF A $\beta_{1-42}$ (Figure 3E), CSF $A \beta_{1-42 / 1-40}$ ratio (Figure 3F), CSF t-tau (Figure 3G), CSF p-tau/t-tau ratio (Figure 3H), CSF $\mathrm{NfL}$ (Figure 3l), CSF p-NfH (Figure 3J), and serum NfL (Figure 3K) were significantly correlated with $\mathrm{mRS}$ on discharge $(P=0.037, P=0.047, P=0.012, P=0.004, P=0.002, P=0.002$, and $P=0.007$, respectively). The correlation coefficient of the association between CSF t-tau and mRS on discharge was positive (i.e., high t-tau levels associated with poor prognosis). Consequently, the correlation coefficient between the CSF p-tau/t-tau ratio (equivalent to reciprocal of CSF t-tau) and $\mathrm{mRS}$ on discharge was negative. CSF NfL, CSF pNfH and serum NfL were also positively associated with a poor prognosis. On the other hand, CSF $A \beta_{1-42}$ and the CSF $A \beta_{1-42 / 1-40}$ ratio were negatively correlated with the $m R S$ scores (i.e., low $A \beta_{1-42}$ levels and $A \beta_{1-42 / 1-40}$ ratio associated with poor prognosis). The significant correlation between CSF NfL and $\mathrm{mRS}$ on discharge was only preserved after age adjustment $(P=0.018)$ among those biomarkers, similar to the case in GCS.

\section{Discussion}

The current study generated three major findings: First, regarding $A \beta$ and tau- related biomarkers, the HSV/VZV group showed significantly lower CSF levels of $A \beta 1-42, A \beta 1-40$, and the $A \beta 1-42 / A \beta 1-40$ ratio compared with the control group, while CSF t-tau and p-tau were significantly elevated in the HZV/VZV group. On comparing HSV and VZV groups, elevation of p-tau was marked in the VZV group and, while the levels of CSF t-tau were elevated specifically in the HSV group. These results correspond to previous reports showing significantly decreased $A \beta 1-42$ [16], increased t-tau, and increased p-tau in CSF of patients with HSV encephalitis $[16,17]$. CSF t-tau elevation is also consistent with changes in CSF t-tau of patients with human herpes virus 6 encephalitis [18] and, therefore, can be considered to reflect neuronal damage due to viral infection [17]. To our best knowledge, this is the first report of CSF p-tau elevation in patients with CNS VZV infection. This suggests that the biomarker profile of decreased A $11-$ 42, increased t-tau, and increased p-tau in CSF might be shared not only by CNS involvement of HSV infection but also CNS VZV involvement. This combination of biomarker changes, the so-called "AD signature", has been considered to indicate the presence of AD pathology. In particular, high levels of p-tau at threonine181 have been reported to occur solely in AD and not in other neurodegenerative disorders[19]. Moreover, CSF p-tau has been reported to remain unchanged even in the presence of acute brain damage, such as acute brain infarction, whilst CSF t-tau shows marked elevation, possibly because p-tau does not simply reflect neuronal damage or degeneration [19]. CSF p-tau elevation in the HSV/VZV group might be attributed to hyperphosphorylation of tau induced by APP mis-metabolism caused by herpetic infection [20], as similarly seen in the case of AD. However, it should be noted that the mis-metabolism of APP leading to decreased CSF $A \beta_{1-42}$ and ${ }_{1-40}$ in CNS herpetic infection is not identical to that in AD [21] [22]. Actually, HSV infection leads to decreased concentrations of both sAPPa and $\beta$ fragments in CSF, unlike in AD patients [16]. Aside from the mechanism, we would like to emphasize the following: the fact that the biomarker profile in AD patients mimics that in patients with CNS HSV and VZV infections should be paid attention to as a confounding factor in the CSF biomarker-based diagnosis, where patients with recent reactivation of HSV or VZV might be misdiagnosed as preclinical AD.

Second, CSF STREM2, a marker reflecting the extent of microglial inflammation, and GFAP, a marker of astrocyte damage, were elevated in the HSV and VZV groups compared with the control group. Of note, these trends are also identical to those reported as biomarker changes in patients with AD [23] [24] [25] [26]. To our knowledge, this is the first report of increased levels of CSF sTREM2 in patients with herpes virus infections. This finding is in line with 
previous observations of elevated CSF STREM2 in patients with autoimmune inflammatory neurological diseases [27] and in HIV-1-infected patients in whom elevated CSF sTREM2 was suggested to reflect microglial activation triggered by viral infection [28]. CNS HSV and VZV infections may lead to the elevation of CSF sTREM2 via a similar mechanism. Regarding GFAP, there have been several reports of increased CSF GFAP in patents with herpes virus infections including HSV-1 encephalitis, Ramsay-Hunt syndrome, and VZV encephalitis, suggesting that astrocytic damage is also present in patients with herpes virus infections [29] [30] [31]. The results of the present study are consistent with those reports.

Third, only NfL levels were significantly correlated with the severity and a poor outcome after age adjustment in CSF biomarkers, while relationships between other biomarkers in CSF and those clinical symptoms disappeared after age adjustment if they were univariately significant. This suggests that the NfL concentration in CSF obtained for the diagnostic purpose on admission is the most powerful as a predictive marker of the severity and prognosis in patients with herpes virus infections among the molecules tested in this study. NfL is a neuronal cytoplasmic protein highly expressed in large-caliber myelinated axons. Thus, CSF levels of this marker are considered to represent the degree of large myelinated axonal damage [32]. Therefore, our results could be interpreted as follows: the severity and prognosis associated with HSV and VZV infections of the CNS depend on the degree of impairment of large myelinated fibers. On the other hand, a few studies reported contradictory results whereby there was no correlation between the CSF NfL concentration and prognosis or neurological sequelae in patients with RamsayHunt syndrome and VZV infection of the CNS [30] [31]. This inconsistency may be due to the difference in the participants: we enrolled not only patients with VZV but also HSV encephalitis, which is generally associated with more marked neurological dysfunction and a poorer outcome than VZV infection. The correlation between CSF and blood NfL levels is reported to be tight in neurological disorders [33] [34]. In fact, serum levels of NfL were strongly associated to their matching CSF levels in our sample set (Supplementary Figure) and consequently, ability of serum NfL to evaluate the severity and to predict prognosis were nearly equivalent to those of CSF NfL when we consider reduced statistical power due to some missing serum samples. Serum NfL might have potential as an efficient and useful blood-based biomarker for prediction of the severity and prognosis.

We acknowledge that the small sample size was a major limitation of this study. Furthermore, the short follow-up period may have weakened the statistical power to detect an association between the prognosis and biomarkers. In the future, it will be necessary to conduct large-scale case-control studies and prospective observations in order to validate the clinical significance of AD-related biomarkers in patients with CNS HSV and VZV infections.

\section{Conclusions}

This study demonstrated significantly decreased CSF $A \beta_{1-42}, A \beta_{1-40}$, and $A \beta_{1-42} / A \beta_{1-40}$ ratio and increased t-tau and p-tau in the HSV/VZV group. In addition, CSF sTREM2, a marker reflecting the extent of microglial inflammation, and GFAP, a marker of astrocytic damage, were elevated in the HSV/VZV group compared with the control group. The CSF profile of decreased $A \beta_{1-42}$ and increased t-tau and p-tau is known as the "AD signature". In particular, elevated CSF p181-tau was recognized as reflecting the presence of AD pathology. Moreover, increased sTREM2 and GFAP in CSF are also identical to those reported as biomarker changes in patients with AD. The fact that the biomarker profile in patients with CNS HSV and VZV infections mimics that in AD patients should be paid attention to as a pitfall in CSF biomarker-based diagnosis of AD. CSF NfL levels were significantly correlated with the disease severity and a poor outcome after age adjustment. The CSF NfL concentration on admission may be useful as a predictive marker of the severity and prognosis in patients with CNS HSV and VZV infections. 


\section{Declarations}

\section{Acknowledgements}

Not applicable

\section{Authors contribution}

F. K-M., T.O. and Y.F. assisted with patient enrollment, data analysis, and interpretation. H.T., F.K-M., and M.S. performed laboratory work and data analysis. D.A. and T.M. participated in review and revision of the manuscript. M.S., T.K. and T.T were involved with conceptualization and design of the study, patient enrollment, data collection, interpretation of the data, and review of the manuscript. All authors reviewed the drafts and approved the final version of the manuscript.

\section{Conflict of financial interests:}

The authors have no competing financial interests. No non-financial conflicts of interest exist for any of the authors

\section{Funding}

This work was mainly supported in part by a grant from the Japan Agency for Medical Research and Development (AMED) (to T.T.), and partially by Grants-in-Aid (Nos. 15K09319 and 18K07506 to TK; No. 20K16605 to T.0.; No. $18 \mathrm{~K} 15461$ to HT) from the Ministry of Education, Culture, Sports, Science and Technology of Japan to T.K, and research grants from Dainippon Sumitomo Pharma in 2018 (to T.O.) and 2017 (to T.K.).

\section{Availability of data and materials}

All data generated or analysed during this study are included in this published article and its supplementary information files.

\section{Ethics approval and consent to participate}

Written informed consent from the participants was obtained when possible and, if not, from the nearest relative. The study protocols were approved by the University Ethics Committee (ERB-G-12, Kyoto Prefectural University of Medicine, Kyoto, Japan).

\section{Consent for publication}

Not applicable

\section{Competing interests}

The authors have no competing financial interests. Also, no non-financial conflicts of interest exist.

\section{Abbreviations}

CNS: central nervous system, AD: Alzheimer's disease, HSV: herpes simplex virus, VZV: varicella zoster virus, $A \beta$ : amyloid $\beta$, t-tau: total tau, $\mathrm{p}$-tau: tau phosphorylated at threonine 181, $\mathrm{NfL}$ : neurofilament light chain, $\mathrm{p}-\mathrm{NfH}$ : phosphorylated neurofilament heavy chain, GFAP: glial fibrillary acidic protein, sTREM2: soluble triggering receptor expressed on myeloid cells 2, CSF: cerebrospinal fluid, MRI: magnetic resonance imaging, mRS: modified Rankin 
Scale, GCS: Glasgow coma scale, T2WI: T2-weighted imaging (T2WI), FLAIR: fluid-attenuated inversion recovery imaging.

\section{References}

1. Blennow K, Dubois B, Fagan AM, Lewczuk P, de Leon MJ, Hampel H: Clinical utility of cerebrospinal fluid biomarkers in the diagnosis of early Alzheimer's disease. Alzheimer's \& Dementia 2015, 11:58-69.

2. Olsson B, Lautner R, Andreasson U, Ohrfelt A, Portelius E, Bjerke M, Holtta M, Rosen C, Olsson C, Strobel G, et al: CSF and blood biomarkers for the diagnosis of Alzheimer's disease: a systematic review and meta-analysis. Lancet Neurol 2016, 15:673-684.

3. Shaw LM, Vanderstichele H, Knapik-Czajka M, Clark CM, Aisen PS, Petersen RC, Blennow K, Soares H, Simon A, Lewczuk P, et al: Cerebrospinal fluid biomarker signature in Alzheimer's disease neuroimaging initiative subjects. Ann Neurol 2009, 65:403-413.

4. Skillback T, Farahmand BY, Rosen C, Mattsson N, Nagga K, Kilander L, Religa D, Wimo A, Winblad B, Schott JM, et al: Cerebrospinal fluid tau and amyloid-beta1-42 in patients with dementia. Brain 2015, 138:2716-2731.

5. Bateman RJ, Xiong C, Benzinger TL, Fagan AM, Goate A, Fox NC, Marcus DS, Cairns NJ, Xie X, Blazey TM, et al: Clinical and biomarker changes in dominantly inherited Alzheimer's disease. N Engl J Med 2012, 367:795-804.

6. Roe CM, Fagan AM, Grant EA, Hassenstab J, Moulder KL, Maue Dreyfus D, Sutphen CL, Benzinger TL, Mintun MA, Holtzman DM, Morris JC: Amyloid imaging and CSF biomarkers in predicting cognitive impairment up to 7.5 years later. Neurology 2013, 80:1784-1791.

7. Itzhaki RF, Lin WR, Shang D, Wilcock GK, Faragher B, Jamieson GA: Herpes simplex virus type 1 in brain and risk of Alzheimer's disease. Lancet 1997, 349:241-244.

8. Lovheim H, Gilthorpe J, Johansson A, Eriksson S, Hallmans G, Elgh F: Herpes simplex infection and the risk of Alzheimer's disease: A nested case-control study. Alzheimers Dement 2015, 11:587-592.

9. Tzeng N-S, Chung C-H, Lin F-H, Chiang C-P, Yeh C-B, Huang S-Y, Lu R-B, Chang H-A, Kao Y-C, Yeh H-W, et al: Antiherpetic Medications and Reduced Risk of Dementia in Patients with Herpes Simplex Virus Infections-a Nationwide, Population-Based Cohort Study in Taiwan. Neurotherapeutics 2018, 15:417-429.

10. Tsai M-C, Cheng W-L, Sheu J-J, Huang C-C, Shia B-C, Kao L-T, Lin H-C: Increased risk of dementia following herpes zoster ophthalmicus. PLoS One 2017, 12:e0188490.

11. Chen VC, Wu SI, Huang KY, Yang YH, Kuo TY, Liang HY, Huang KL, Gossop M: Herpes Zoster and Dementia: A Nationwide Population-Based Cohort Study. J Clin Psychiatry 2018, 79.

12. Suárez-Calvet $M$, Kleinberger $G$, Araque Caballero $M$, Brendel $M$, Rominger A, Alcolea $D$, Fortea J, Lleó $A$, Blesa R, Gispert JD, et al: sTREM2 cerebrospinal fluid levels are a potential biomarker for microglia activity in early-stage Alzheimer's disease and associate with neuronal injury markers. EMBO Mol Med 2016, 8:466-476.

13. Tunkel AR, Glaser CA, Bloch KC, Sejvar JJ, Marra CM, Roos KL, Hartman BJ, Kaplan SL, Scheld WM, Whitley RJ: The management of encephalitis: clinical practice guidelines by the Infectious Diseases Society of America. Clin Infect Dis 2008, 47:303-327.

14. Tatebe H, Kasai T, Ohmichi T, Kishi Y, Kakeya T, Waragai M, Kondo M, Allsop D, Tokuda T: Quantification of plasma phosphorylated tau to use as a biomarker for brain Alzheimer pathology: pilot case-control studies including patients with Alzheimer's disease and down syndrome. Mol Neurodegener 2017, 12:63.

15. Ohara T, Hata J, Tanaka M, Honda T, Yamakage H, Yoshida D, Inoue T, Hirakawa Y, Kusakabe T, Shibata M, et al: Serum Soluble Triggering Receptor Expressed on Myeloid Cells 2 as a Biomarker for Incident Dementia: The 
Hisayama Study. Ann Neurol 2019, 85:47-58.

16. Krut JJ, Zetterberg H, Blennow K, Cinque P, Hagberg L, Price RW, Studahl M, Gisslen M: Cerebrospinal fluid Alzheimer's biomarker profiles in CNS infections. J Neurol 2013, 260:620-626.

17. Di Stefano A, Alcantarini C, Atzori C, Lipani F, Imperiale D, Burdino E, Audagnotto S, Mighetto L, Milia MG, Di Perri G, Calcagno A: Cerebrospinal fluid biomarkers in patients with central nervous system infections: a retrospective study. CNS Spectr 2019:1-7.

18. Tanuma N, Miyata R, Nakajima K, Okumura A, Kubota M, Hamano S, Hayashi M: Changes in cerebrospinal fluid biomarkers in human herpesvirus-6-associated acute encephalopathy/febrile seizures. Mediators Inflamm 2014, 2014:564091.

19. Blennow K, Zetterberg H: Biomarkers for Alzheimer's disease: current status and prospects for the future. $J$ Intern Med 2018, 284:643-663.

20. Civitelli L, Marcocci ME, Celestino I, Piacentini R, Garaci E, Grassi C, De Chiara G, Palamara AT: Herpes simplex virus type 1 infection in neurons leads to production and nuclear localization of APP intracellular domain (AICD): implications for Alzheimer's disease pathogenesis. J Neuroviro/ 2015, 21:480-490.

21. Shipley SJ, Parkin ET, Itzhaki RF, Dobson CB: Herpes simplex virus interferes with amyloid precursor protein processing. BMC Microbiol 2005, 5:48.

22. Cairns DM, Rouleau N, Parker RN, Walsh KG, Gehrke L, Kaplan DL: A 3D human brain-like tissue model of herpes-induced Alzheimer's disease. Science Advances 2020, 6:eaay8828.

23. Suárez-Calvet M, Araque Caballero MÁ, Kleinberger G, Bateman RJ, Fagan AM, Morris JC, Levin J, Danek A, Ewers M, Haass C: Early changes in CSF sTREM2 in dominantly inherited Alzheimer's disease occur after amyloid deposition and neuronal injury. Sci Transl Med 2016, 8:369ra178-369ra178.

24. Oeckl P, Halbgebauer S, Anderl-Straub S, Steinacker P, Huss AM, Neugebauer H, von Arnim CAF, Diehl-Schmid J, Grimmer T, Kornhuber J, et al: Glial Fibrillary Acidic Protein in Serum is Increased in Alzheimer's Disease and Correlates with Cognitive Impairment. J Alzheimers Dis 2019, 67:481-488.

25. Ishiki A, Kamada M, Kawamura Y, Terao C, Shimoda F, Tomita N, Arai H, Furukawa K: Glial fibrillar acidic protein in the cerebrospinal fluid of Alzheimer's disease, dementia with Lewy bodies, and frontotemporal lobar degeneration. J Neurochem 2016, 136:258-261.

26. Abu-Rumeileh S, Steinacker P, Polischi B, Mammana A, Bartoletti-Stella A, Oeckl P, Baiardi S, Zenesini C, Huss A, Cortelli $\mathrm{P}$, et al: CSF biomarkers of neuroinflammation in distinct forms and subtypes of neurodegenerative dementia. Alzheimers Res Ther 2019, 12:2.

27. Piccio L, Buonsanti C, Cella M, Tassi I, Schmidt RE, Fenoglio C, Rinker J, 2nd, Naismith RT, Panina-Bordignon P, Passini N, et al: Identification of soluble TREM-2 in the cerebrospinal fluid and its association with multiple sclerosis and CNS inflammation. Brain 2008, 131:3081-3091.

28. Gisslén M, Heslegrave A, Veleva E, Yilmaz A, Andersson LM, Hagberg L, Spudich S, Fuchs D, Price RW, Zetterberg H: CSF concentrations of soluble TREM2 as a marker of microglial activation in HIV-1 infection. Neurol Neuroimmunol Neuroinflamm 2019, 6:e512.

29. Grahn A, Hagberg L, Nilsson S, Blennow K, Zetterberg H, Studahl M: Cerebrospinal fluid biomarkers in patients with varicella-zoster virus CNS infections. J Neurol 2013, 260:1813-1821.

30. Lindström J, Grahn A, Zetterberg H, Studahl M: Cerebrospinal fluid viral load and biomarkers of neuronal and glial cells in Ramsay Hunt syndrome. Eur J Neurosci 2016, 44:2944-2949. 
31. Studahl M, Rosengren L, Günther G, Hagberg L: Difference in pathogenesis between herpes simplex virus type 1 encephalitis and tick-borne encephalitis demonstrated by means of cerebrospinal fluid markers of glial and neuronal destruction. J Neurol 2000, 247:636-642.

32. Neurofilament light chain as a biomarker in neurological disorders. Journal of Neurology, Neurosurgery \& Psychiatry 2019, 90:870-881.

33. Gisslén M, Price RW, Andreasson U, Norgren N, Nilsson S, Hagberg L, Fuchs D, Spudich S, Blennow K, Zetterberg $\mathrm{H}$ : Plasma Concentration of the Neurofilament Light Protein (NFL) is a Biomarker of CNS Injury in HIV Infection: A Cross-Sectional Study. EBioMedicine 2016, 3:135-140.

34. Kasai T, Kojima Y, Ohmichi T, Tatebe H, Tsuji Y, Noto YI, Kitani-Morii F, Shinomoto M, Allsop D, Mizuno T, Tokuda T: Combined use of CSF NfL and CSF TDP-43 improves diagnostic performance in ALS. Ann Clin Transl Neurol 2019.

\section{Tables}

Table 1 Characteristics of the participants.

A:

\begin{tabular}{|c|c|c|c|c|c|c|c|c|c|c|c|}
\hline Case & Sex & Age & Diagnosis & $\begin{array}{l}\text { CSF } \\
\text { PCR }\end{array}$ & $\begin{array}{l}\text { CSF } \\
\text { HSV } \\
\text { IgM }\end{array}$ & $\begin{array}{l}\text { Serum } \\
\text { HSV } \\
\text { IgM }\end{array}$ & $\begin{array}{l}\text { CSF } \\
\text { cell } \\
\text { count } \\
(/ \mu \mathrm{L})\end{array}$ & $\begin{array}{l}\text { CSF } \\
\text { protein } \\
(\mathrm{mg} / \mathrm{dL})\end{array}$ & $\begin{array}{l}\text { Grade } \\
\text { of MRI } \\
\text { finding }\end{array}$ & GCS & $\mathrm{mRS}$ \\
\hline $1^{*}$ & $M$ & 52 & Encephalitis & $(-)$ & $(-)$ & $(+)$ & 28 & 60 & 1 & 13 & 2 \\
\hline $2^{*}$ & M & 45 & Encephalitis & $(-)$ & $(-)$ & $(-)$ & 99 & 62 & 4 & 15 & 0 \\
\hline $3^{*}$ & $M$ & 56 & Encephalitis & $(-)$ & $(-)$ & $(+)$ & 26 & 77 & 2 & 14 & 3 \\
\hline $4^{*}$ & $\mathrm{~F}$ & 22 & Encephalitis & $(+)$ & $(+)$ & $(+)$ & 62 & 37 & 3 & 11 & 2 \\
\hline $5^{\star}$ & $\mathrm{F}$ & 66 & Encephalitis & $(+)$ & N/A & N/A & 24 & 83 & 4 & 13 & 3 \\
\hline $6^{*}$ & $\mathrm{~F}$ & 56 & Myelitis & $(-)$ & $\mathrm{N} / \mathrm{A}$ & $(+)$ & 7 & 30 & $N / A^{*}$ & 15 & 2 \\
\hline 7 & $\mathrm{~F}$ & 33 & Meningitis & $\mathrm{N} / \mathrm{A}$ & $(+)$ & $(-)$ & 364 & 155 & 1 & 15 & 0 \\
\hline 8 & $F$ & 47 & Meningitis & $\mathrm{N} / \mathrm{A}$ & $\mathrm{N} / \mathrm{A}$ & $(+)$ & 38 & 59 & 1 & 15 & 0 \\
\hline $9^{*}$ & $M$ & 83 & Encephalitis & $(+)$ & $\mathrm{N} / \mathrm{A}$ & $(+)$ & 249 & 271 & 2 & 3 & 4 \\
\hline
\end{tabular}

B: 


\begin{tabular}{|lllllllllllll|}
\hline Case & Sex & Age & Diagnosis & $\begin{array}{l}\text { CSF } \\
\text { PCR }\end{array}$ & $\begin{array}{l}\text { CSF } \\
\text { VZV } \\
\text { IgM }\end{array}$ & $\begin{array}{l}\text { Serum } \\
\text { VZV } \\
\text { ggM }\end{array}$ & $\begin{array}{l}\text { CSF } \\
\text { cell } \\
\text { count } \\
(/ \mu \mathrm{L})\end{array}$ & $\begin{array}{l}\text { CSF } \\
\text { protein } \\
(\mathrm{mg} / \mathrm{dL})\end{array}$ & $\begin{array}{l}\text { MRI } \\
\text { finding }\end{array}$ & GCS & mRS \\
\hline 1 & M & 32 & Myelitis & $(-)$ & $(-)$ & $(+)$ & $3^{\#}$ & $\begin{array}{l}28 \\
\text { Medulla } \\
\text { oblongata to } \\
\text { C2 }\end{array}$ & 15 & 2 \\
\hline $2^{*}$ & F & 84 & Meningitis & $(-)$ & $(-)$ & $(+)$ & 99 & 129 & N/A & 14 & 3 \\
\hline 3 & M & 21 & Meningitis & $(+)$ & N/A & N/A & 91 & 54 & N/A & 15 & 0 \\
\hline $4^{*}$ & F & 27 & Meningitis & $(+)$ & N/A & N/A & 303 & 80 & N/A & 14 & 0 \\
\hline 5 & M & 70 & Meningitis & $(+)$ & N/A & N/A & 95 & 206 & N/A & 15 & 1 \\
\hline $6^{*}$ & F & 15 & Meningitis & $(+)$ & N/A & N/A & 1312 & 114 & N/A & 15 & 0 \\
\hline 7 & M & 55 & Encephalitis & $(+)$ & N/A & N/A & 660 & 193 & N/A & 6 & 0 \\
\hline 8 & F & 71 & Meningitis & N/A & N/A & N/A & 5 & 32 & N/A & 15 & 0 \\
\hline & & & & & & & & & & & \\
\hline
\end{tabular}

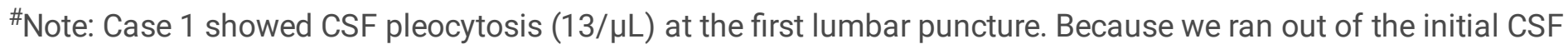
sample, we used the sample collected 3 days after the first examination.

C: 


\begin{tabular}{|c|c|c|c|c|c|}
\hline Case & Sex & Age & Diagnosis or symptom & CSF cell count $(/ \mu \mathrm{L})$ & CSF protein (mg/dL) \\
\hline $1^{*}$ & $\mathrm{~F}$ & 42 & Influenza & 6 & 38 \\
\hline 2 & $\mathrm{~F}$ & 25 & Fever elevation & 12 & 25 \\
\hline 3 & M & 29 & Fever elevation & 36 & 30 \\
\hline $4^{*}$ & $\mathrm{~F}$ & 72 & Crowned dens syndrome & 6 & 39 \\
\hline 5 & $\mathrm{~F}$ & 36 & Fever elevation & 21 & 32 \\
\hline $6^{*}$ & M & 71 & Cervical spondylosis & 6 & 49 \\
\hline 7 & $\mathrm{~F}$ & 73 & Neuralgic amyotrophy & 9 & 39 \\
\hline 8 & M & 50 & Cervical spondylosis & 12 & 187 \\
\hline 9 & M & 66 & Fatigue & 3 & 35 \\
\hline 10 & M & 77 & Oculomotor paresis & 3 & 42 \\
\hline $11^{*}$ & M & 67 & Dysphagia & 3 & 56 \\
\hline 12 & M & 44 & Viral myositis & 3 & 36 \\
\hline 13 & $\mathrm{~F}$ & 63 & Transient global amnesia & 0 & 19 \\
\hline $14^{*}$ & $M$ & 44 & Psychophysiologic disorder & 9 & 28 \\
\hline $15^{*}$ & $M$ & 37 & Peroneal nerve paralysis & 6 & 33 \\
\hline 16 & $\mathrm{~F}$ & 49 & Fever elevation & 3 & 24 \\
\hline 17 & $M$ & 59 & Brachial plexus neuropathy & 3 & 36 \\
\hline 18 & $\mathrm{~F}$ & 24 & Guillain-Barré syndrome & 24 & 29 \\
\hline
\end{tabular}

Clinical characteristics of the HSV (A), VZV (B), and control (C) groups are shown.

Cases who also measured serum NfL were indicated by asterisks. The degree of MRI involvement in the HSV group was categorized into the following five grades: Grade 1: no apparent abnormality on MRl; Grade 2: unilateral medial temporal abnormality on T2-weighted imaging (T2WI) or fluid-attenuated inversion recovery imaging (FLAIR); Grade 3: unilateral imaging abnormality on T2WI or FLAIR spreading from the medial to lateral temporal lobe; Grade 4: unilateral imaging abnormality on T2WI or FLAIR spreading from the temporal lobe to other lobes except for the temporal lobe; Grade 5: bilateral imaging abnormality on T2WI or FLAIR. *: case 6 was not categorized because of the lack of brain involvement (spinal cord involvement at Th 2-4). GCS showed the lowest score during hospitalization. The scores of mRS are those at discharge. N/A: not available.

Table 2 Regression analyses of clinical status and biomarkers. 


\begin{tabular}{|c|c|c|c|c|}
\hline & \multicolumn{4}{|c|}{ Lowest score of GCS } \\
\hline & \multicolumn{2}{|c|}{ Univariate analyses } & \multicolumn{2}{|c|}{ Multivariate analyses (age-adjusted) } \\
\hline & $\rho:$ & P: & $\mathrm{B} \pm \mathrm{SE}$ & $P:$ \\
\hline $\operatorname{CSF} A \beta_{1-42}$ & 0.131 & 0.618 & & \\
\hline $\operatorname{CSF} A \beta_{1-40}$ & 0.063 & 0.811 & & \\
\hline $\operatorname{CSF~A} \beta_{1-42} / A \beta_{1-40}$ & 0.190 & 0.465 & & \\
\hline CSF t-tau & -0.814 & $<0.001^{\star \star}$ & $-0.168 \times 10^{-3} \pm 0.0910 \times 10^{-3}$ & 0.065 \\
\hline CSF p-tau & -0.274 & 0.287 & & \\
\hline CSF p-tau /t-tau & 0.257 & 0.319 & & \\
\hline CSF NfL & -0.583 & $0.014^{*}$ & $-0.325 \times 10^{-3} \pm 0.132 \times 10^{-3}$ & $0.014^{*}$ \\
\hline CSF p-NfH & $-0.534^{*}$ & $0.027 *$ & $-0.162 \times 10^{-3} \pm 0.0900 \times 10^{-3}$ & 0.071 \\
\hline CSF sTREM2 & -0.332 & 0.194 & & \\
\hline CSF GFAP & -0.443 & 0.075 & & \\
\hline \multirow[t]{4}{*}{ Serum NfL } & -0.804 & $0.007^{\star \star}$ & $-0.48 \pm 0.022$ & $0.030 *$ \\
\hline & \multicolumn{4}{|c|}{$\mathrm{mRS}$ at discharge } \\
\hline & \multicolumn{2}{|c|}{ Univariate analyses } & \multicolumn{2}{|c|}{ Multivariate analyses (age-adjusted) } \\
\hline & $\rho:$ & P: & $\mathrm{B} \pm \mathrm{SE}$ & P: \\
\hline $\operatorname{CSF} A \beta_{1-42}$ & -0.508 & $0.037^{*}$ & $-2.051 \times 10-3 \pm 1.436 \times 10-3$ & 0.161 \\
\hline $\operatorname{CSF} A \beta_{1-40}$ & -0.334 & 0.189 & & \\
\hline $\operatorname{CSF} A \beta_{1-42} / A \beta_{1-40}$ & -0.488 & $0.047^{\star}$ & $-20.539 \pm 13.9$ & 0.142 \\
\hline CSF t-tau & $0.592^{*}$ & $0.012^{*}$ & $-0.173 \times 10^{-3} \pm 0.0966 \times 10^{-3}$ & 0.073 \\
\hline CSF p-tau & -0.303 & 0.237 & & \\
\hline CSF p-tau /t-tau & -0.652 & $0.004^{\star \star}$ & $-1.273 \pm \quad 0.708$ & 0.072 \\
\hline CSF NfL & 0.683 & $0.002^{\star \star}$ & $0.373 \times 10^{-3} \pm 0.158 \times 10^{-3}$ & $0.018^{*}$ \\
\hline CSF p-NfH & 0.691 & $0.002^{\star \star}$ & $0.014 \times 10^{-3} \pm 0.009 \times 10^{-3}$ & 0.129 \\
\hline CSF sTREM2 & 0.163 & 0.531 & & \\
\hline CSF GFAP & 0.404 & 0.108 & & \\
\hline Serum NfL & 0.812 & $0.007 \star \star$ & $0.065 \pm 0.041$ & 0.108 \\
\hline
\end{tabular}

Result of uni- and multivariate regression analyses of the biomarkers and GCS/mRS are shown. "*”: $P<0.05$. “**”:P<0.01 
Figures
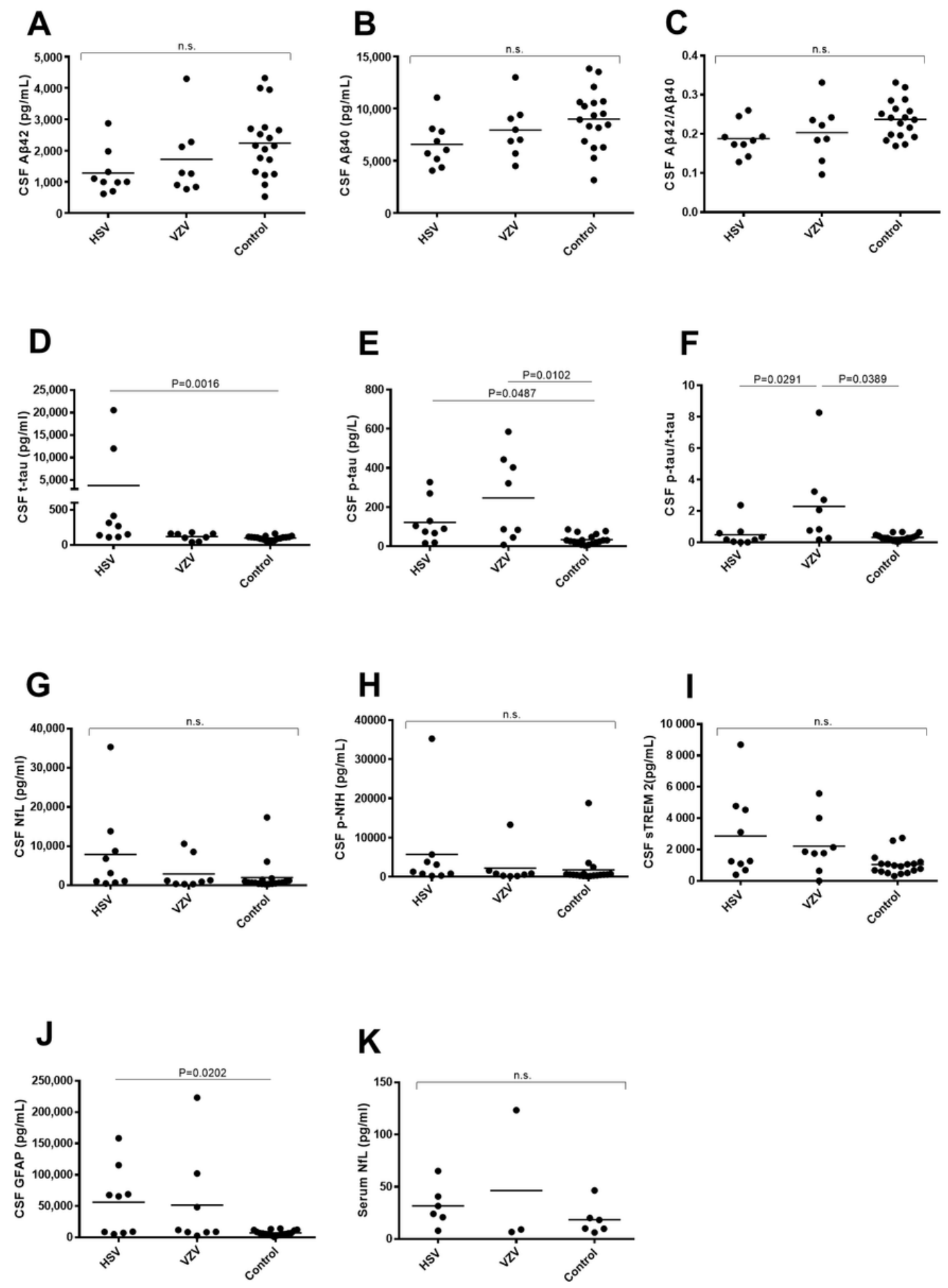

Figure 1

Comparison of biomarker concentrations among the HSV, VZV, and control groups ( $A$ : CSF A $1-42$, B: CSF A 1 1-40, C: CSF AB1-42/1-40 ratio, D: CSF t-tau, E:CSF p-tau, F: CSF p-tau/t-tau ratio, G: CSF NfL, H: CSF pNfH, l: CSF STREM2, J: CSF GFAP, and K: serum NfL). The CSF (HSV group: $n=9$, VZV group: $n=8$, control group: $n=18$ ) and serum (HSV group: $n=6, V Z V$ group: $n=3$, control group: $n=6$ ) levels of those biomarkers in each individual are shown as a black circle. Bars indicate median values. When multiple comparisons failed to reach significance, "n.s." (not significant) was placed on $\mathrm{n}$-shaped zig-zag lines. When a significant difference was observed among the 
groups, P-values in the subsequent post-hoc analyses were placed on the simple lines. (Unless otherwise specified, P-values on multiple comparisons were omitted.)

A

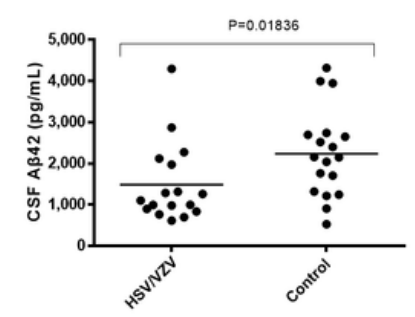

D

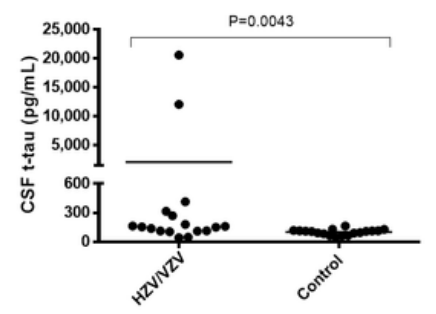

G

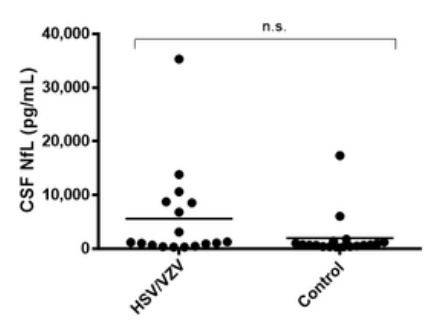

J

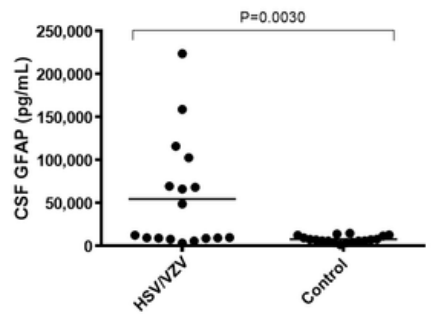

B

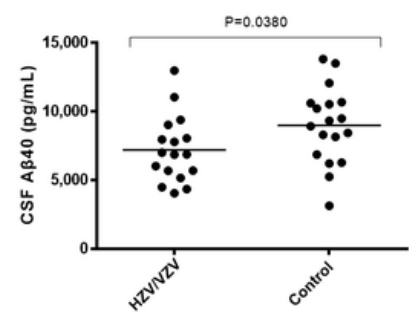

E
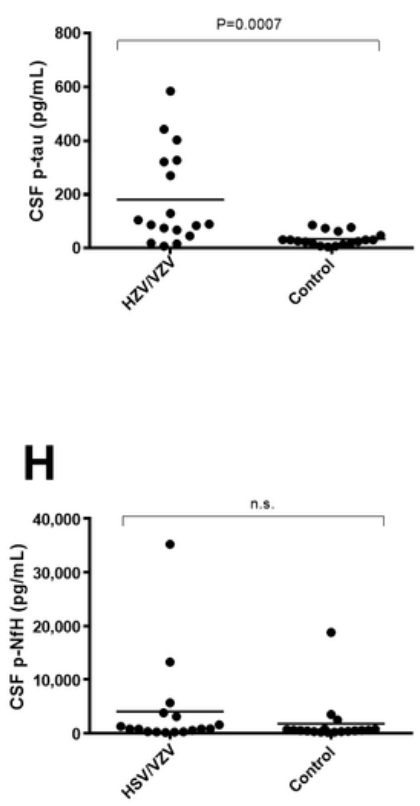

K

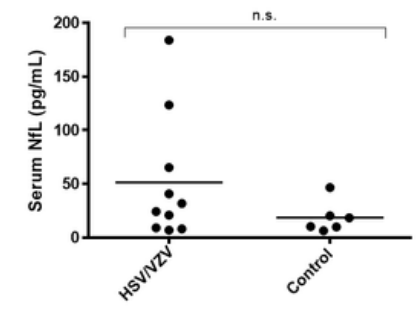

C

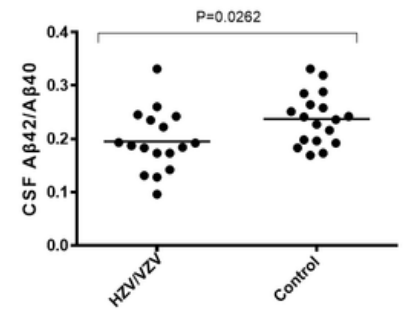

$\mathbf{F}$
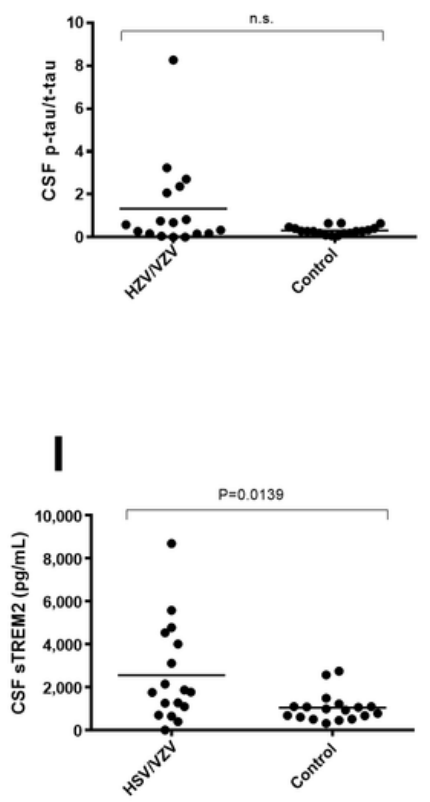

\section{Figure 2}

Comparison of biomarker concentrations between the HSV/VZV and control groups( A: CSF A 1 1-42, B: CSF A $1-40$, C: CSF Aß1-42/1-40 ratio, D: CSF t-tau, E:CSF p-tau, F: CSF p-tau/t-tau ratio, G: CSF NfL, H: CSF pNfH, I: CSF sTREM2, J: CSF GFAP, and K: serum NfL). The CSF (HSV/VZV group: $n=17$; control: $n=18$ ) and serum (HSV/VZV group: $n=9$; control: $n=6$ ) levels of those biomarkers in each individual are shown as a black circle. Bars indicate median values. When a significant difference was observed between the groups, P-values were placed on n-shaped zig-zag lines. "n.s." indicates "not significant". 

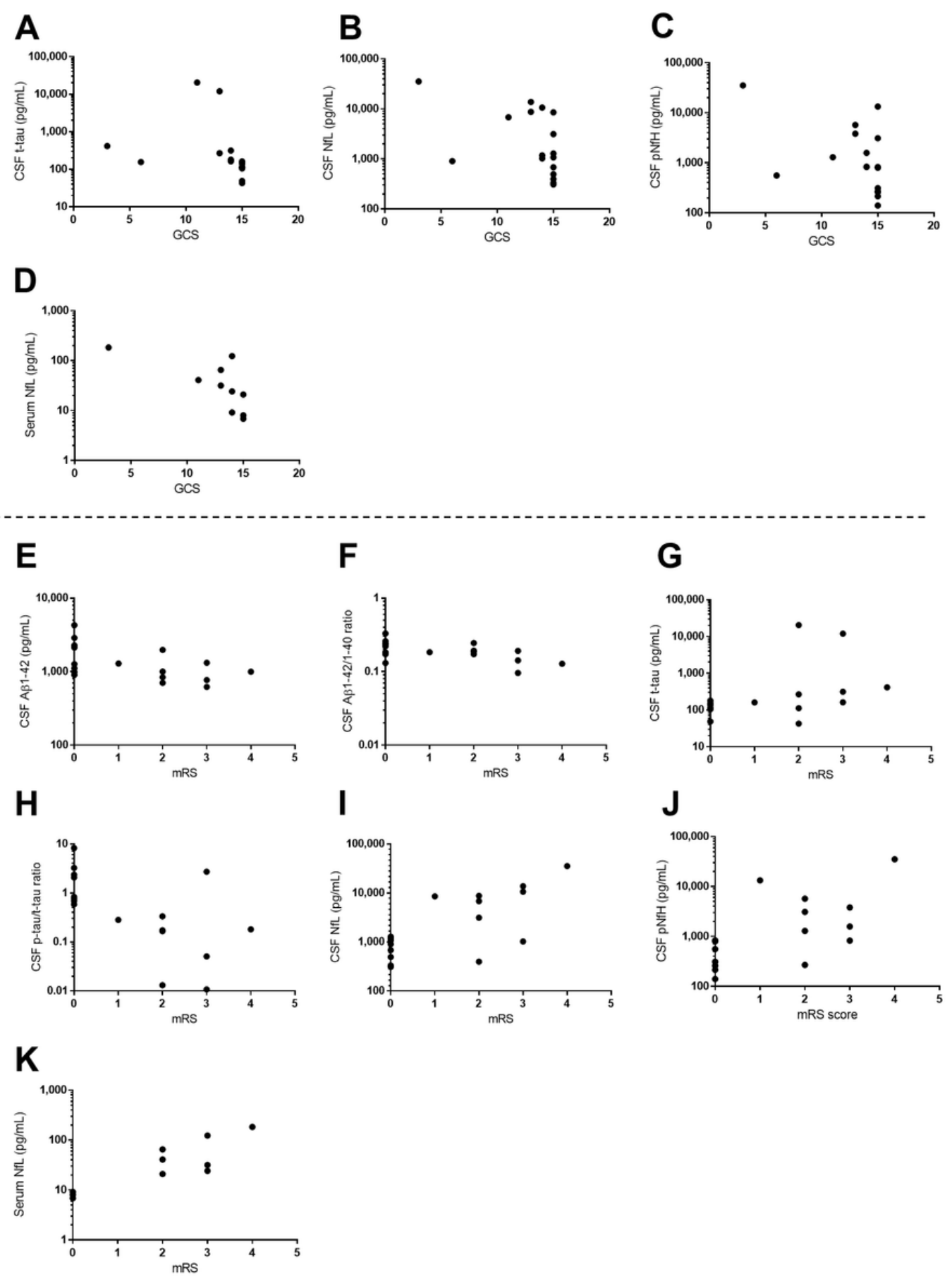

\section{Figure 3}

Scatter plots of the biomarkers vs. the lowest score of GCS during the hospitalization $(A, B, C, D)$ as well as the biomarkers vs. $m R S$ score at discharge $(E, F, G, H, I, J, K)$ in the HSV/VZV group. Note: graphs only shown when there was significant correlation on univariate correlation analysis. A: CSF t-tau vs. GCS, B: CSF NfL vs. GCS, C: CSF pNfH

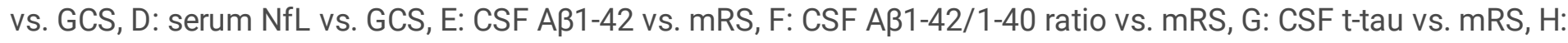
CSF p-tau/t-tau ratio vs. mRS, I: CSF NfL vs. mRS, J: CSF pNfH vs. mRS, K: serum NfL vs. mRS). Y-axes of graphs show concentrations of biomarkers using a logarithmic scale. X-axes represent GCS or mRS scores.

\section{Supplementary Files}


This is a list of supplementary files associated with this preprint. Click to download.

- Supplementary2020819.docx 